\title{
FGF23 and bone microarchitecture
}

\author{
T. Rupp ${ }^{1} \cdot$ M. Amling ${ }^{1} \cdot$ T. Rolvien ${ }^{1,2}$
}

Received: 31 July 2019 / Accepted: 1 August 2019 / Published online: 12 August 2019

(C) International Osteoporosis Foundation and National Osteoporosis Foundation 2019

We thank Dr. Minisola and coworkers for their interest in our article entitled "High FGF23 levels are associated with impaired trabecular bone microarchitecture in patients with osteoporosis" [1], in which we have outlined the significant association between high c-terminal FGF23 (cFGF23) and impaired bone microarchitecture.

Next to normal FGF23 levels in most of the included osteoporosis patients, we noticed moderately increased FGF23 levels in a number of patients without phosphate wasting. In fact, we treat several patients with confirmed tumor-induced osteomalacia (TIO) by ${ }^{68} \mathrm{Ga}$ DOTA-TATE PET/CT and very high FGF23 levels > $1000 \mathrm{kRUL} / \mathrm{l}$ in our department, but it is correct that there are other patients with TIO and only moderately increased or normal FGF23 levels [2]. While we had already excluded two patients with TIO in the initial phase of the study, we indeed included also osteoporosis patients with FGF23 values well above the specified reference range. In the patient with the highest FGF23 level, we repeatedly observed low phosphate values. Extended diagnostics including ${ }^{68} \mathrm{Ga}$ DOTA-TATE PET/CT and genetic screening for all known mutations leading to increased FGF23 levels (e.g., PHEX) were all inconspicuous. However, we are aware that these results do not necessarily exclude TIO. The patient with the second highest FGF23 level showed no laboratory signs of phosphate wasting.

In accordance with the present commentary by Minisola et al., we have now performed the same statistical analyses after exclusion of these two patients with the highest FGF23 levels. All associations between cFGF23 and trabecular microarchitecture remained significant in both the correlation analysis and the multiple linear regression model, besides trabecular number at the distal tibia (correlation analysis: $p=$

T. Rolvien

t.rolvien@uke.de

1 Department of Osteology and Biomechanics, University Medical Center Hamburg-Eppendorf, Lottestr. 59, 22529 Hamburg, Germany

2 Department of Orthopedics, University Medical Center Hamburg-Eppendorf, Hamburg, Germany
0.114). Interestingly, the correlation analysis yielded an additional significant negative association between FGF23 and cortical BMD at the distal tibia $(p=0.047)$.

It is still debated which FGF23 assay (especially cFGF23 vs. intact FGF23 (iFGF23)) is the most appropriate to detect certain disease states of phosphate metabolism. While iFGF23 appears to be more biologically relevant, it was found that cFGF23 assays showed lower intra-individual variation [3]. As we had recently identified cases, where iFGF23 had failed to identify patients clearly presenting with clinical and laboratory signs of phosphate wasting, we decided to perform this study using the cFGF23 assay. Nonetheless, iFGF23 measurements might be superior in other cases, and this needs to be explored further.

Lastly, we appreciate the authors' similar view on the potential direct role of FGF23 in bone mineralization, which is indeed supported by previous mouse studies including those using $\mathrm{Hyp}$ and $\mathrm{Npt} 2^{-/}$mice. Although there is certainly a high complexity underlying the pleiotropic actions of FGF23, there is evidence for a negative influence of FGF23 on mineralization, potentially explained by FGF23-mediated suppression of the tissue-nonspecific alkaline phosphatase [4].

\section{References}

1. Rupp T, Butscheidt S, Vettorazzi E, Oheim R, Barvencik F, Amling M, Rolvien T (2019) High FGF23 levels are associated with impaired trabecular bone microarchitecture in patients with osteoporosis. Osteoporos Int 30:1655-1662

2. Breer S, Brunkhorst T, Beil FT, Peldschus K, Heiland M, Klutmann S, Barvencik F, Zustin J, Gratz KF, Amling M (2014) 68Ga DOTATATE PET/CT allows tumor localization in patients with tumorinduced osteomalacia but negative $111 \mathrm{In}$-octreotide SPECT/CT. Bone 64:222-227

3. Smith ER, Cai MM, McMahon LP, Holt SG (2012) Biological variability of plasma intact and C-terminal FGF23 measurements. J Clin Endocrinol Metab 97:3357-3365

4. Erben RG (2017) Pleiotropic actions of FGF23. Toxicol Pathol 45: 904-910

Publisher's note Springer Nature remains neutral with regard to jurisdictional claims in published maps and institutional affiliations. 